\title{
Single Top quark production cross section using the ATLAS detector at the LHC
}

\author{
Caterina Monini* \\ on behalf of the ATLAS collaboration \\ LPSC, Université Grenoble-Alpes, CNRS/IN2P3 \\ E-mail: monini@lpsc.in2p3.fr
}

\begin{abstract}
We present single top quark production measurements stemming from the analysis of proton proton collision data collected by the ATLAS detector at the LHC. The fiducial and total inclusive $t$-channel cross sections, as well as the $W t$ associated production cross section are determined with the full $8 \mathrm{TeV}$ dataset, while the top quark-antiquark ratio in the $t$-channel is extracted using $4.7 \mathrm{fb}^{-1}$ data at $7 \mathrm{TeV}$. All the observed values, as well as the $\left|V_{t b}\right|$ element of the CabibboKobayashi-Maskava matrix directly measured within those analyses, are in agreement with the Standard Model predictions. We discuss, in addition, the limits set on exotic single top production at $8 \mathrm{TeV}$; this includes the search for flavor changing neutral currents in the $t$-channel, and for heavy $W^{\prime}$ resonances in the s-channel.
\end{abstract}

XXII. International Workshop on Deep-Inelastic Scattering and Related Subjects, 28 April - 2 May 2014

Warsaw, Poland

\footnotetext{
* Speaker.
} 


\section{Introduction}

At the LHC, top quarks are generated in pairs via strong interaction and, with a lower rate, via charged-current electroweak interaction mediated by a $W$ boson. According to the regime of the latter, such single top events are sorted into three distinct mechanisms, whose Feynman diagrams are reported in Fig. 1 and approximate NNLO cross sections in Table 1: the $t$-channel exchange of a space-like $W$ boson 1(a), the associated production of an on-shell $W$ boson and a top quark 1(b), the $s$-channel production involving the decay of a time-like $W$ boson 1(c).

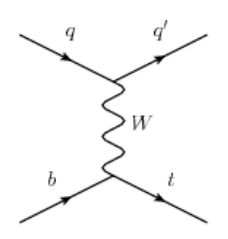

(a)

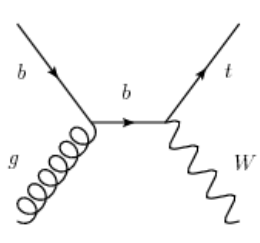

(b)

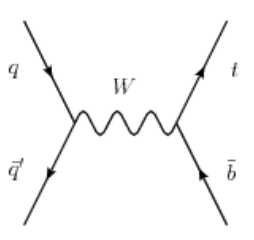

(c)

Figure 1: LO Feynman diagrams for single top (a) $t$-channel, (b) $W t$ and (c) $s$-channel productions.

\begin{tabular}{c|c|c}
\hline \hline & LHC @ 7 TeV & LHC @ 8 TeV \\
\hline \hline$\sigma_{t}$ & $64.57_{-1.74}^{+2.63} \mathrm{pb}$ & $87.76_{-1.91}^{+3.44} \mathrm{pb}$ \\
$\sigma_{W t}$ & $15.74_{-1.21}^{+1.17} \mathrm{pb}$ & $22.37_{-1.52}^{+1.52} \mathrm{pb}$ \\
$\sigma_{s}$ & $4.63_{-0.18}^{+0.20} \mathrm{pb}$ & $5.61_{-0.21}^{+0.21} \mathrm{pb}$ \\
\hline
\end{tabular}

Table 1: Single top cross sections at $\sqrt{s}=7$ and $8 \mathrm{TeV}$, based on NLO+NNLL calculations [1].

Top quark electroweak production is interesting as it provides a direct probe of the $W-t-b$ vertex and is, as well, highly sensitive to new physics. The following document presents the latest results achieved with the analysis of proton-proton collisions with the ATLAS detector [4], interpreted in terms of the Standard Model and Beyond Standard Model (BSM) predictions. In order to avoid redundancy, an overview of the objects definition and multivariate techniques which are shared by all the single top analyses will be presented in the first section.

\section{Common objects definition and signal discrimination}

The top quark decays almost at 100\% into a $b$ quark and a $W$ boson, that may then undergo either a hadronic or a leptonic decay; the former, however, is neglected in order to endure the overwhelming LHC multijet background. Single top events are therefore selected via single-lepton triggers, and characterized by the additional presence of jets and a neutrino. Electron candidates, reconstructed by means of a cluster-based algorithm, are required to belong to the pseudorapidity region $\left|\eta_{\mathrm{cl}}\right|<$ 2.47 (with the calorimeter barrel-endcap transition region excluded) and to have $p_{\mathrm{T}}>25 \mathrm{GeV}$. Muon candidates are obtained by combining the muon spectrometer hits with the inner detector tracks, with $p_{\mathrm{T}}>25 \mathrm{GeV}$ and $|\eta|<2.5$. Both kinds of leptons fulfill tight identification criteria, and need as well to be isolated from other physics objects [2], [3]. Jets are reconstructed using the anti- $k_{t}[5]$ algorithm with a radius parameter of 0.4 , correcting the detector response by $p_{\mathrm{T}^{-}}$ 
and $\eta$-dependent factors to provide an average energy scale calibration. Specific requirements are applied in order to reduce the contamination from pile-up events and misidentification effects. Jets, for which in general a $p_{\mathrm{T}}$ thresholds of $30 \mathrm{GeV}$ is required, can be tagged as arising from Bhadrons via neural network-based algorithms which exploit the specific features of bottom quarks, i.e. long lifetime and significant flight path leading to measurable secondary vertices. Finally, the missing transverse momentum $\left(E_{\mathrm{T}}^{\mathrm{miss}}\right)$ measures the imbalance due to the escaping neutrino, whose longitudinal component is derived by applying the $W$ boson pole-mass constraint.

Several sources of background may mimic this signature, featuring event topology and kinematics similar to single top events. Multivariate techniques are employed in order to disentangle such processes from the signal, which in general is produced at very low rates. Two alternative methods are Boosted Decision Trees (BDT), based on a "forest" of binary classifiers that implement a sequential selection by cutting on the variable that provides, at each step, the best signalbackground separation, and Neural Networks $(\mathrm{NN})$ which use Bayesian regularization techniques for the training phase to improve generalization performance and avoid overtraining.

\section{Standard Model precision measurements}

A precise determination of the three single top production mechanisms cross sections is crucial not only to confirm the SM predictions, but also to directly extract the $\left|V_{t b}\right|$ element of the CabibboKobayashi-Maskava matrix and constrain the proton parton density functions (PDFs).

The first measurement of the $t$-channel fiducial cross section has been performed with $20.3 \mathrm{fb}^{-1}$ data collected by the ATLAS detector at $\sqrt{s}=8 \mathrm{TeV}$ [6]. In order to match the final state depicted in Fig. 1(a), events are selected owing to the presence of an isolated electron or muon, two jets (one of which must be b-tagged) and $E_{\mathrm{T}}^{\text {miss }}>30 \mathrm{GeV}$; the additional requirement $m_{\mathrm{T}}(W)>50$ $\mathrm{GeV}$ is applied to suppress the multijet contamination, which is estimated via a data-driven technique as in the other reported analyses. At this stage, a NN classifier is implemented to separate $t$-channel events from the expected background, the dominant contributions from $t \bar{t}$ and $W+$ jets being checked in specific control regions. 14 input variables are thus combined in a discriminant distribution, on which a maximum likelihood fit to the data is performed to extract the signal contribution $(\hat{v})$. Then, in order to minimize the uncertainty related to the theoretical modelling (event generator and parton shoer scheme), the $t$-channel cross section is determined in a fiducial volume within the detector acceptance. Defining $\varepsilon_{s e l}$ as the fraction of selected events to be in the fiducial volume, $\varepsilon_{f i d}$ as the fraction of the fiducial events fulfilling the offline selection, and $\mathscr{L}$ as the integrated luminosity, one can express:

$$
\sigma_{\text {fid }}=\frac{\varepsilon_{\text {sel }}}{\varepsilon_{f i d}} \times \frac{\hat{v}}{\mathscr{L}}
$$

The fiducial cross section for different next-to-leading order (NLO) and leading order (LO) generators is shown in Fig. 2(a); the measured value obtained when $t$-channel events are generated with ACERMC is found to be: $\sigma_{f i d}=3.36 \pm 0.05$ (stat.) \pm 0.48 (syst.) \pm 0.09 (lumi.) pb, where the total relative uncertainty of $\pm 14 \%$ is dominated by jet energy scale $\eta$-intercalibration and $t$-channel generator. Eq. 3.1 allows, then, to extrapolate the total $t$-channel cross section using the acceptance of several generator models and renormalization scales, as shown in Fig. 2(b). 
The inclusive value is in agreement with the NLO+NNLL calculation within the uncertainty and, when $t$-channel events are generated with aMC@NLO, it yields:

$\sigma_{t}=85.8 \pm 1.2$ (stat.) \pm 11.4 (syst.) \pm 3.1 (PDF) \pm 2.3 (lumi.) pb.

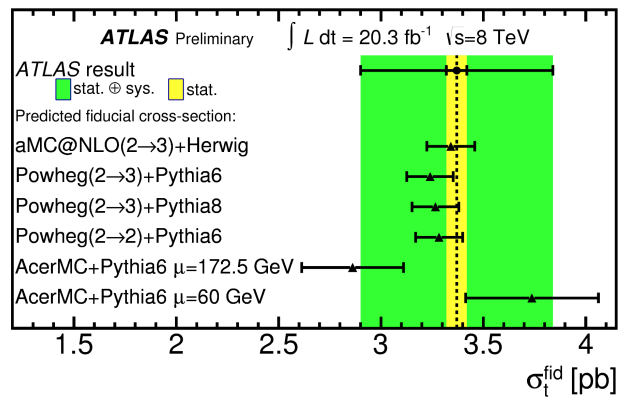

(a)

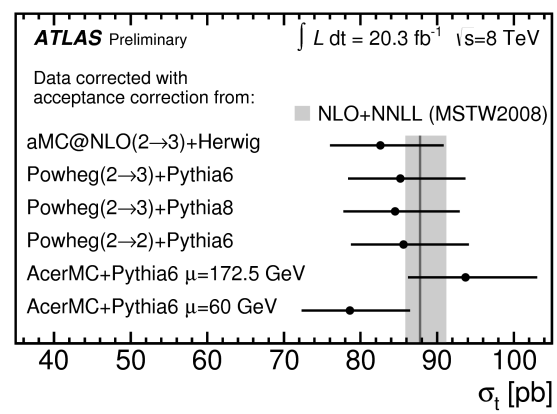

(b)

Figure 2: (a) Predicted $t$-channel fiducial cross section for different MC generators, and measured value. (b) Inclusive $t$-channel cross section obtained by extrapolating (a) to the full phase space [6].

A similar analysis was performed with the full $7 \mathrm{TeV}$ dataset of $4.7 \mathrm{fb}^{-1}$, to determine the $t$-channel single top quark and antiquark ratio $\left(R_{t}\right)$ [7]; the interest of an accurate measurement relies on the fact that this observable is sensitive to the ratio of up to down quarks PDFs in the momentum fraction range $0.02<x<0.5$, and features smaller uncertainties with respect to the single cross sections because of partial cancellations. Almost the same selection requirements as for the analysis detailed above are implemented, but events can contain two or three jets and are sorted into two categories according to the lepton charge; the latter, in fact, allows to determine if the electron or muon arises from the decay of a top quark or antiquark. Four neural networks separately trained in the four selected channels ( 2 and 3 jets-bins, positive and negative lepton) are fitted simultaneously to extract the top quark and antquark ratio. The measured cross section ratio agrees with the NLO predictions using different PDF sets, but suffers from a large uncertainty, dominated by the statistical error, the background normalization and the initial and final state radiations:

$$
R_{t}=1.81 \pm 0.10 \text { (stat.) }{ }_{-0.20}^{+0.21} \text { (syst.). }
$$

A measurement of the $W t$-channel cross section was realized with the full proton-proton collision dataset collected at $\sqrt{s}=8 \mathrm{TeV}$, corresponding to an integrated luminosity of $20.3 \mathrm{fb}^{-1}$ [8]. The analysis is performed with dilepton final states, requiring one isolated electron and one isolated muon with opposite charge, and one or two jets (one of which b-tagged); $W$ boson leptonic decays grant an efficient suppression of the $W+$ jets and multijet backgrounds, and this particular configuration yields the best signal purity. After the event selection, the signal is discriminated by the dominant $t \bar{t}$ background by means of two BDT classifiers, which are trained separately in the 1 and 2 jets-bins in order to account for differences in the topology and kinematics of the two samples. A simultaneous maximum likelihood fit coupled to a frequentist statistical analysis allows to determine the $W t$ single top contribution: $\sigma_{W t}=27.2 \pm 2.8$ (stat.) \pm 5.4 (syst.) pb. The measurement, which features a significance of 4.2 standard deviations, is in agreement with the SM predictions within its total relative uncertainty of $\pm 21.3 \%$; the main contributions to the latter derive from the theory modelling, the jet energy scale and the b-tagging efficiency. 
Single top measurements are especially valuable for the extraction of $\left|V_{t b}\right|$, since both the production and decay mechanisms are described in terms of a $W-t-b$ vertex. Such a matrix element is indeed directly assessed as the ratio of measured to predicted cross section, assuming $V_{t b} \gg$ $V_{t d}, V_{t s}$ and left-handed weak coupling. With no hypothesis on the number of fermions generations nor on the unitarity of the CKM matrix, $\left|V_{t b}\right|$ is found to be $0.97_{-0.10}^{+0.09}$ using the observed $t$-channel cross section and yields $1.10 \pm 0.12$ (exp.) \pm 0.03 (theory) with the $W t$ cross section, where both values are determined within the $8 \mathrm{TeV}$ analyses.

\section{Searches for Beyond Standard Model physics}

The top quark electroweak production mechanisms are separately sensitive to a variety of beyond Standard Model scenarios, that can hence be constrained by comparing the three measured cross sections. At the same time, single top constitutes a fruitful context to undertake direct modelindependent searches for specific new physics manifestations.

Several BSM models predict enhanced Flavour Changing Neutral Current (FCNC) rates, which are instead highly suppressed within the Standard Model. A search for $t$-channel single top production via the FCNC process $q g \rightarrow t \rightarrow l v b$ has been thus carried out, triggered by the good sensitivity and the unique kinematical properties of such signature. $14.2 \mathrm{fb}^{-1}$ of ATLAS data at $\sqrt{s}=8 \mathrm{TeV}$ have been analyzed [9] following the strategy detailed in Section 3 for the $t$-channel cross section measurement, but requiring only one jet, b-tagged. The strong FCNC events, simulated with a NLO generator, are isolated from background with a $\mathrm{NN}$ discriminant which is fitted using a Bayesian statistical approach. Since no excess is observed in data, as Fig. 3(a) illustrates, an upper limit of $\sigma_{q g \rightarrow t} \times \mathscr{B}(t \rightarrow b W)<2.5 \mathrm{pb}$ is set at 95\% CL. This is as well interpreted in terms of limits on the coupling constants and branching ratios of the processes $t \rightarrow c g$ and $t \rightarrow u g$, in two dimensions.

An analysis based on $14.3 \mathrm{fb}^{-1}$ of ATLAS data at $\sqrt{s}=8 \mathrm{TeV}$ [10] aims at identifying new heavy charged gauge bosons $\left(W^{\prime}\right)$, that can potentially be detected via hadronic decay $W^{\prime} \rightarrow t b \rightarrow l v b b$. Such a search is particularly important to complement the analyses in the leptonic channels, since it is sensitive to leptophobic $W^{\prime}$ models and is extended to candidates of both chiralities. In order to match the expected s-channel signature depicted in Fig. 1(c), the event selection is based on slightly different criteria than the nominal ones: one isolated electron or muon is required with $p_{\mathrm{T}}>30 \mathrm{GeV}$, with two or three central jets (among which two b-tagged) with $p_{\mathrm{T}}>25 \mathrm{GeV}$ and $E_{\mathrm{T}}^{\text {miss }}>35 \mathrm{GeV}$; the so-called triangular cut $E_{\mathrm{T}}^{\text {miss }}+m_{\mathrm{T}}(W)>60 \mathrm{GeV}$ is also implemented to suppress the contamination from multijet production. Futhermore, since the simulation is known to provide a poor modelling for the $W+$ jets sample, which represents a significant background contribution (second only to top quark pair), a data-driven scale factor is estimated in a specific control region to correct its normalization. To extract the $W^{\prime}$ cross section, two BDT classifiers are trained in the 2 and 3 jets-bins, considering as signal a right-handed heavy resonance with $m_{W^{\prime}}=1.75 \mathrm{TeV}$. The BDT algorithm is then applied to signal samples accounting for different chiralities and masses in the range $(0.5,3.0) \mathrm{TeV}$. As no excess is found (one particular case is depicted in Fig. 3(b)), limits at the 95\% CL exclude the existence of $W_{L}^{\prime}\left(W_{R}^{\prime}\right)$ with masses below $1.74(1.84) \mathrm{TeV}$; further constraints are as well derived for the coupling constant $g^{\prime}$. 


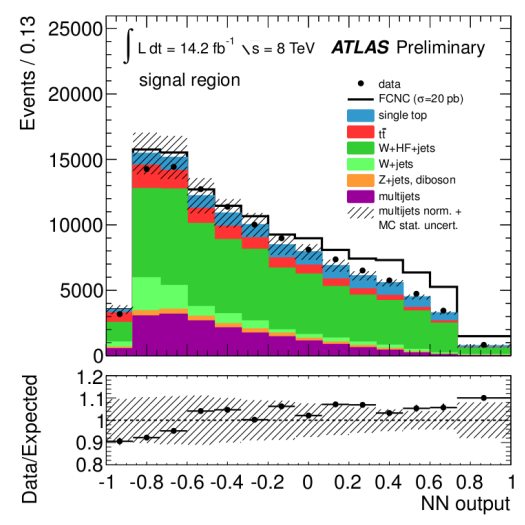

(a)

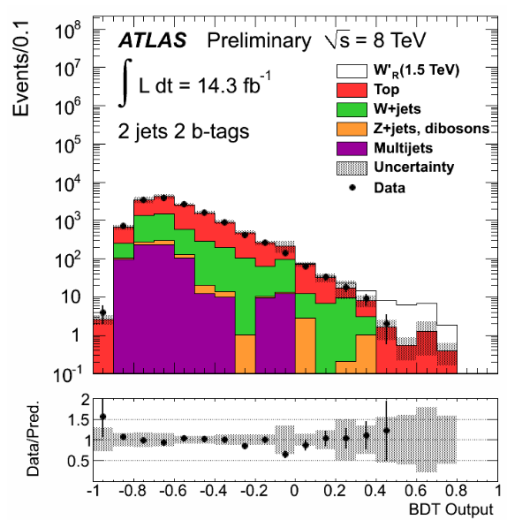

(b)

Figure 3: (a) NN output distribution with FCNC signal shape stacked on top of the backgrounds and scaled to $20 \mathrm{pb}$ [9]. (b) BDT output distribution in the 2 jets-bin, with the contribution of a particular $W^{\prime}$ boson with $m_{W_{R}^{\prime}}=1.5 \mathrm{TeV}$ appearing on the right [10].

\section{Summary}

This document, far from giving a complete overview of the studies performed by the ATLAS collaboration on the single top production, reports the most recent results which stem from the analysis of $p p$ collisions at $\sqrt{s}=7$ and $8 \mathrm{TeV}$. The measured cross sections for the $t$-channel and $W t$, as well as the $\left|V_{t b}\right|$ element of the CKM matrix directly extracted from these measurements, are compatible with the SM predictions. The cross section ratio of top quark and antiquark in the $t$-channel has not yet the needed sensitivity for setting further constraints on the up to down quarks ratio in the proton. Finally, exclusion limits are set on a strong FCNC process and on the production of a heavy $W^{\prime}$ resonance involving single top final states.

\section{References}

[1] N.Kidonakis, Phys. Rev. D 83 091503(R) (2011), Phys. Rev. D 82, 054018 (2010), Phys. Rev. D 81, 054028 (2010).

[2] ATLAS Collaboration, ATL-COM-PHYS-2012-1197, https://cds.cern.ch/record/1472525 .

[3] ATLAS Collaboration, ATL-COM-PHYS-2013-1016, https://cds.cern.ch/record/1563201 .

[4] ATLAS Collaboration, 2008 JINST 3 S08003.

[5] M. Cacciari, G.P. Salam, G. Soyez, JHEP 080463 (2008).

[6] ATLAS Collaboration, ATLAS-CONF-2014-007, https://cds.cern.ch/record/1668960 .

[7] ATLAS Collaboration, ATLAS-CONF-2012-056, https://cds.cern.ch/record/1453783 .

[8] ATLAS Collaboration, ATLAS-CONF-2013-100, https://cds.cern.ch/record/1600799 .

[9] ATLAS Collaboration, ATLAS-CONF-2013-063, https://cds.cern.ch/record/1562777 .

[10] ATLAS Collaboration, ATLAS-CONF-2013-050, https://cds.cern.ch/record/1547566 . 